Página inicial: 97 - Página final: 106

Tipo de artículo: de investigación

\title{
Sazón y formación: prácticas alimenticias e identidad cultural en las familias afrodescendientes de la comuna ocho de Medellin ${ }^{7}$.
}

Seasoning and training: nutritional practices and cultural identity in people of african descent families of the commune eight of Medellin.

Recibido: mayo de 2015 Revisado: octubre de 2015 Aceptado: noviembre 20 de 2015

Por: Jair Hernando Álvarez Torres ${ }^{1}$, Deisy Marcela Cifuentes Ortiz ${ }^{2}$, Yessica Isabel Isaza Puerta ${ }^{3}$, Lina María Idárraga Pérez ${ }^{4}$, Manuela Giraldo Zuluaga ${ }^{5}$ y Diana Patricia Zapata Hernández ${ }^{6}$.

\section{Resumen.}

Con el presente artículo se pretende construir una ruta hermenéutica que genere un acercamiento teórico frente a los cambios en las prácticas alimenticias de algunas familias ubicadas en la comuna ocho de Medellin, para comprender sus condiciones de posibilidad en la comunidad afrodescendiente que por factores personales, grupales, culturales, políticos o económicos, se han visto obligados a migrar a un contexto diferente al suyo, donde sus prácticas y estilos de vida han sufrido algunas modificaciones. En ese orden de ideas, se abordaron conceptos tales como prácticas alimenticias, familias afrodescendientes, migración y acción social que permitieron dar claridad y comprensión al tema de interés.

\section{Palabras clave.}

Prácticas Alimenticias, Acción Social, Familias Afrodescendientes, Migración.

\begin{abstract}
.
This article is intended to build a hermeneutic path that generates a theoretical approach to changes in the nutritional practices of some families located in the Commune Eight of Medellin, in order to understand its conditions of possibility in the community of African descent, who for personal, group, cultural, political, or economic factors have been forced to migrate to a context different from theirs where its practices and lifestyles have undergone some changes. Along these lines, concepts such as nutritional practices, families, people of African descent, migration, and social action, that allowed you to give clarity and understanding to the topic of interest, were addressed.
\end{abstract}

\section{Key words.}

Nutritional practices; Social action; Afro-descendant families; and Migration.

\footnotetext{
${ }^{1}$ Doctor en Historia (Universidad Nacional de Colombia), Estudios Doctorales en Historia (Universidad Torcuato Di Tella, Argentina) Licenciado en Filosofia (Universidad de Antioquia). Docente investigador del Grupo Interdisciplinario de Estudios Pedagógicos (GIDEP), Línea Educación y Cultura Visual, Universidad de San Buenaventura, seccional Medellín (Colombia). Contacto: jair.alvarez777@gmail.com

${ }^{2}$ Licenciada en Educación Preescolar, Facultad de Educación, Universidad de San Buenaventura Seccional Medellin (Colombia). Contacto: deisymcifuentes@hotmail.com

${ }^{3}$ Licenciada en Educación Preescolar, Facultad de Educación, Universidad de San Buenaventura Seccional Medellín (Colombia). Joven investigadora. Contacto: isabelisaza89@gmail.com

${ }^{4}$ Licenciada en Educación Preescolar, Facultad de Educación, Universidad de San Buenaventura Seccional Medellín. Joven investigadora. Contacto: linamip1223@hotmail.com

${ }^{5}$ Licenciada en Educación Preescolar, Facultad de Educación, Universidad de San Buenaventura Seccional Medellin. Joven Investigadora. Contacto: manu418@hotmail.com

${ }^{6}$ Licenciada en Educación Preescolar, Facultad de Educación, Universidad de San Buenaventura Seccional Medellín. Joven Investigadora. Contacto: dipazahe@hotmail.com
} 


\section{Introducción.}

El presente artículo hace parte del macroproyecto "Prácticas de crianza escolares, familiares y comunitarias: un diálogo de saberes con las familias de la Asociación de Mujeres Afrodescendientes de Medellín (AMAM) y de la Fundación las Golondrinas de la Comuna Ocho", desarrollado por la Universidad de San Buenaventura, la Corporación Universitaria Lasallista y la Fundación Las Golondrinas.

En este sentido a partir del proyecto sobre las prácticas de crianza surge el interés por indagar las prácticas alimenticias de la población afrodescendiente, para comprender sus condiciones de posibilidad en la comunidad afrodescendiente que por factores politicos, sociales y económicos, se han visto obligados a migrar a un contexto diferente al suyo, donde sus prácticas y estilos de vida han sufrido algunas modificaciones culturales importantes.

De esta manera, se parte de la familia como el primer espacio socializador del ser humano, donde se fundan y se construyen un sin número de valores, costumbres, hábitos y prácticas, dando paso a la formación identitaria a partir de las prácticas alimenticias y con ellas a factores de regulación dentro y fuera de la familia, constituyendo a partir del medio como alimento, mediaciones como la educación de sus integrantes, los roles, la configuración de las jerarquías a nivel familiar y su vinculación con los procesos culturales adyacentes.

En este orden de ideas, se retoman las prácticas alimenticias, ya que el alimento además de ser una necesidad básica y fisiológica para el ser humano, es un generador de espacios socializadores y diferenciadores de culturas, debido a que traen consigo un valor ancestral y una carga cultural en la que se resaltan los modos de adquisición, preparación, y consumo caracterizados por los sabores y olores como símbolos de identidad, o lo que es lo mismo, se generan a partir de la alimentación, procesos formativos o constitución de subjetividades.

Desde esta perspectiva, la investigación tiene una mirada pedagógica en la que se inició con una búsqueda teórica que fundamentó y mostró las prácticas alimenticias en comunidades afrodescendientes, para luego pasar a lo práctico, en donde se realizó un trabajo de campo, desde el paradigma cualitativo, con un enfoque hermenéutico, y se utilizó como herramienta el estudio de caso, con dos familias que permitieron sustentar las transformaciones en sus respectivas prácticas alimenticias.

\section{Desarrollo del tema.}

Para hablar de prácticas alimenticias, se hace necesario de manera inicial, definir "prácticas" que según Foucault, y citado por Álvarez Torres; Pemberty Sepúlveda; Blandón Giraldo; Grajales Crespo (2012, p.91) son entendidas como:

La racionalidad o la regularidad que organiza lo que los hombres hacen (sistemas de acción en la medida en que están habitados por el pensamiento), que tiene un carácter sistemático (saber, poder, ética) y general (recurrente), y que por ello constituye una 'experiencia' o un 'pensamiento'. 
En este sentido, las prácticas se convierten en los pensamientos materializados que regulan los comportamientos y conductas que el ser humano tiene en su vida cotidiana. Ahora bien, en relación con las prácticas alimenticias, Bourdieu (1988) citado por Enrique (2004) las concreta como uno de "los pocos ámbitos donde las clases populares definen un arte de vivir, basado en una moral de la buena vida" (167).

De acuerdo a lo anterior, las prácticas alimenticias son una forma de comprender el estilo de vivir de una población, considerando los alimentos como una muestra de la herencia cultural, tanto a la hora de cultivarlos, obtenerlos, prepararlos, como servirlos e ingerirlos.

Durante el trabajo de campo se conversaron con varias mujeres adultas frente a estos temas, y sus apreciaciones fueron muy importantes para el desarrollo de este trabajo. De este modo, la Mujer Adulta 1 (Para proteger la privacidad de las personas que participaron en el estudio de caso, se han reservado sus nombres y su identidad, generando una codificación sencilla, a modo de serie, donde se alude a las mujeres participantes así: Mujer Adulta 1 y Mujer Adulta 2). comenta la importancia de cultivar productos en su huerta, lo cual demuestra la habilidad de conocer y dominar la tierra en cuestiones de cultivos haciendo de esta práctica una posibilidad de alimentarse y hacer de las comidas únicas y especiales. Así lo mencionó cuando expresó que el cilantro de su huerta era especial, donde si bien no dijo cuál era su especialidad, expresó que poseía menos químicos, dando a entender que los alimentos cultivados en su huerta por sus propias manos y cuidado, son de su preferencia para cocinar.

La huerta en su lugar de origen era un espacio que les posibilitaba cultivar y producir sus propios alimentos, gracias al terreno con el que contaban. En la actualidad, son pocas las personas que luego del proceso migratorio logran tener una huerta en su casa o cerca de ella por diversos factores, uno de ellos tiene que ver con "que le echan mucha basura", así lo explicó la Mujer Adulta 2, quien se queja de la contaminación ambiental en el lugar donde vive, debido al tratamiento indebido de las basuras de las casas en su vecindario; pero a la vez, se queja de la forma como los productos alimenticios están cargados de sustancias químicas artificiales y dañinas para las personas. En ese sentido, la huerta se convierte en un medio facilitador de alimentos, que se pierde o se transforma al llegar a la ciudad de Medellín, debido a las condiciones precarias de vivienda y la contaminación existente, dejando de ser un facilitador de alimentos y de condimentos.

Por otro lado, es pertinente señalar que, una de las constantes de la vida humana que alcanza mayor persistencia en el estilo de vida de cada población en particular, está representada por el patrón dietético, es decir, por la combinación compleja de alimentos que dan forma a una dieta, el cual representa un medio de expresión del ser humano (Barragán, 2005, p.133). De esta manera, los alimentos tienen una relevante asociación cultural en cuanto a prácticas, actitudes y creencias relacionadas con dicho patrón dietético.

Dicha afirmación es compartida por la Mujer Adulta 1, quien considera que, más allá de si está en buen estado o preparado el terreno para el cultivo lo que predomina en esta comunidad es el significado y la creencia que le tienen a la a las fases de la luna, a propósito de sus huertas, pues sembrarlo en tal temporada depende el resultado del cultivo. Con esto entonces, se ve que prevalece el tiempo lunar para este tipo de prácticas más que el estado del terreno. 
En la entrevista a profundidad sostenida con la Mujer Adulta 1 y la Mujer Adulta 2, se llegó a un aspecto importante en cuanto a la regularidad de la alimentación, pues la intención era indagar por los momentos, las horas y las formas de alimentarse durante el transcurso del día, y en esa medida, sus respuestas encontraron la siguiente interpretación por parte del equipo investigador al momento de la depuración de la información: en cuanto a los horarios de alimentación haciendo una comparación entre sus hábitos en su lugar de origen y los hábitos actuales en el lugar donde viven, se encontró que se conservan costumbres como alimentarse tres veces al día (desayuno, almuerzo y comida) aunque no es de obligatorio cumplimiento consumir alguna de estas comidas.

Así pues, la temporalidad no es prioritaria, pero se tiene la experiencia por parte de la Mujer Adulta 1, de que hay que tener algo de comer mientras están las comidas oficiales del dia. "El juguito" parece una alternativa para mitigar el hambre y de esta manera, tolerar la situación de ausencia de comida. Es posible que este hábito se haya adquirido desde su hogar materno, aunque no necesariamente por ausencia de comida. Precisamente, con relación a "el juguito" y su preparación, hace referencia al líquido ingerido durante el día y en las diferentes comidas oficiales de la entrevistada, se hace visible una preferencia por cocinar las frutas para elaborar el jugo, lo anterior tiene su razón de ser, debido a que en el pasado no contaban con los medios para hacerlo de otra forma y ahora, ella considera que preparar algunas frutas de esta forma propicia un mejor sabor en su resultado.

En efecto, el ser humano constituye su subjetividad, entre otras cosas, por la herencia cultural ofrecida por la familia, y en ese sentido, el tema de la alimentación no es la excepción, pues se pudo entrever las preferencias por algunos alimentos poseedores de significados que rememoran momentos, personas o lugares. Como lo manifiesta la Mujer Adulta 2 cuando dijo: “iAhh! el que más me acuerda el Urabá es como cuando uno hacía el pescao frito y patacón, el arroz con coco y la chicha de carambolo y piña"( Hace referencia a bebida típica del pacífico que puede ser fermentada o no de frutas, maíz y arroz).

En este caso, los alimentos evocan momentos o lugares de su historia de vida, es muy factible que ello suceda, ya que la Mujer Adulta 2 pudo haber vivido momentos significativos en Urabá y porque dentro de la adquisición, la preparación y el consumo de dichos platos en este lugar, se involucraron todos los sentidos que se despiertan actualmente y se conectan con los sentidos y las emociones.

Tajes (2006) define la comida "desde una perspectiva sociocultural, como un ente creador de Otredad y un medio de asimilar, rechazar o relacionarse con el otro" (p.47), es una construcción social y una forma de fundar socialmente al individuo, puesto que se convierte en generador de interacción y espacios donde se comparten las mismas prácticas culturales. Así lo confirmó la Mujer Adulta 1 al decir:

Cuando vienen ellos [los familiares desde su lugar de origen] se reúnen y ponen una cuota y traen muchas cosas y también las traen hechas: pescado aliñado y lo fritamos; cuando no, allá está el asadero de carne y ahí asamos la carne, en fechas así como el 31 de diciembre, el 1 de enero, comimos carne que trajeron, después cocinamos papas al vapor, hicimos pan de yuca para el desayuno y sancocho para el almuerzo (Mujer Adulta 1). 
Desde esta perspectiva, las familias de acuerdo a sus vivencias y contextos van construyendo una identidad que les permite propiciar experiencias significativas para cada uno de sus integrantes como grupo o como miembro particular de un entorno.

Así como lo afirma la cartilla "Familia, relaciones y transformaciones" (2006), la familia es el primer espacio socializador que tiene el ser humano y en ella se dan los primeros aprendizajes, acuerdos y diferencias que se ven reflejados en la interacción social. La familia "es el núcleo fundamental constituido por vínculos naturales o jurídicos y en esta se da el desarrollo de normas, valores símbolos, ideologías e identidades" (Gobernación de Antioquia, 2006, p.4) que se transmiten de generación en generación. Es importante resaltar que en Colombia las familias se han visto obligadas a enfrentar cambios culturales, políticos y sociales que han marcado sus vidas y han transformado sus costumbres.

De este modo, la comida es tomada como acto social, es protagonista en diferentes festividades pues eso pudimos interpretar cuando la Mujer Adulta 1 dijo que cuando su familia se reúne en las fechas especiales preparan diferentes alimentos que se caracterizan por ser y tener mezclas, además, de secretos culinarios de la comida Chocoana y Antioqueña. De esta manera, los alimentos se convierten en una excusa para disfrutar en familia, saborear recetas y evocar momentos.

En cuanto a las familias afrodescendientes y su estructuración, son lejanas a las definiciones de familia convencional que se han dado a lo largo de la historia desde lo legal y lo religioso, puesto que esta comunidad trae consigo una historia de esclavitud cargada de tristeza y dolor que ha influido en su estructuración como familia.

De igual modo, en las entrevistas a profundidad realizadas, las informantes coincidente en que su concepto de familia implica un número extenso de personas. En ese sentido, la familia está compuesta no solo por papá, mamá e hijo, sino que también incluye otros integrantes, tales como abuelos, tíos y primos, bien sea por parte del padre, de la madre o ambos, pues estas comunidades no tienen unas reglas estructuradas para que se unan o se separen, debido a que no establecen juicios de valor para las decisiones de cada persona, para ellos es normal que tanto hombres como mujeres tengan durante su vida varios maridos o mujeres sin importar el motivo bien sea por viudez o separación (De La Torre, 2012, p.124). Cabe resaltar que los vínculos afectivos son entendidos como una relación de cariño y amor recíproco entre diferentes personas. Lo anterior, se pudo analizar durante la entrevista a la Mujer Adulta 1 quien mencionó que la muerte de su esposo dejó un vacío en ella, ya que lo recuerda con nostalgia y tristeza demostrándolo en cada uno de sus comentarios.

En este orden de ideas, la forma en que constituyen sus vínculos afectivos y emocionales con otras personas, permite nombrar su tipología familiar a partir de la categoría de "familia extensa"; ya que para ellos es importante el parentesco simbólico, pues les es sumamente importante la vecindad y el compadrazgo a la hora de hacerlos miembros de la familia; en muchos casos dicen que "el vecino es más que familia, porque en un momento de desesperación es él el primero en tenderle la mano" (De la Torre, 2012, p.130) y por esta razón, los vecinos para este tipo de familias son muy significativos. 
La vecindad se hace evidente cuando de colaboración se trata, y así lo menciona la entrevistada cuando dice "es como muy buen vecino" al expresarse y compartir la experiencia de lo que hizo uno de ellos en su casa. Posiblemente para ella la ayuda brindada por este vecino tiene un gran significado ya que en la cultura afrodescendiente, la familia no necesariamente tiene que ser consanguínea sino también aquellos que sin esperar nada a cambio colaboran y brindan ayuda en diversas situaciones.

Es decir, que cualquiera que sea el vínculo familiar, lo que más prima en estas familias es la ayuda mutua, puesto que no es tan primordial la consanguinidad, si no el tener una buena relación siendo solidarios unos con otros, ya que el ayudar contribuye a la consolidación de una familia.

Es importante retomar que la familia afrodescendiente en su conformación aún se ve muy marcada por el género donde la mujer juega un papel fundamental en la unión y estructuración de la familia, es ella quien ha tenido la oportunidad de pasar más tiempo en casa y en comunidad por las funciones culturales que ha tenido que cumplir. Éstas han estado relacionadas con las labores domésticas y formación de los hijos, en tanto es denominada como "hacedora de comunidad" (De La Torre, 2012, p,120). Por su parte, el hombre en esta población ha cumplido el papel de trabajador, para suplir las necesidades básicas de la familia, incluso esa es una de las causas por las que este género migre primero de su lugar de origen en busca de oportunidades. Por último, en cuanto a los hijos de las comunidades negras, además de ser concebidos como una gran compañía, son quienes desde muy pequeños los comienzan a preparar y formar para que sean seres independientes con el fin de que prontamente contribuyan desde el trabajo, es decir, que según el género cumplan con las funciones culturalmente mencionadas y se valgan por sí solos (De la Torre, 2012).

En cuanto a las labores del hogar, como sucede con la preparación de los alimentos y aunque no compete a esta investigación las tareas de género, es de resaltar que tanto hombres como mujeres están en capacidad de desarrollarlas, ya que se ha procurado la transmisión de conocimientos en su vida cotidiana o en ocasiones su aprendizaje ha sido empírico, pues para los afrocolombianos el arte de cocinar es sinónimo de identidad.

Con lo anterior se pudo entrever durante la entrevista a la Adulta Mayor 1, quien desde su experiencia familiar manifestó que no solo la mujer es vista como el personaje de las labores domésticas, sino que también pueden ser ejecutadas por cualquier miembro de la familia ya sea hombre o mujer, expresiones como "en este tiempo los hombres tienen que saber cocinar", "mi hijo sabe hacer unas tortas deliciosas que yo no sé ", "mi hijo mayor cogía la máquina de coser para estrechar su pantalón él mismo", dejan ver una enculturación familiar en donde el aprendizaje de las labores domésticas son una herramienta para la vida y no un asunto género.

Por su parte, con la información suministrada por la Mujer Adulta 2 al preguntársele si sus hijos le colaboraban en la cocina, se reafirmó lo dicho anteriormente al indicar que como familia tienen la oportunidad de realizar las diferentes labores del hogar en especial cuando se trata de cocinar, actividad que les permite conservar las tradiciones culinarias de generación en generación, y que aunque "toque aprender a los golpes en la cabeza" 
(Expresión que utiliza la entrevistada para indicar que se aprende a la fuerza, con dificultad), cuando se quiere cocinar basta el recordar los gustos, los olores, los sabores y colores para poder preparar un delicioso plato con el toque particular dado por los intengrantes de su cultura afrodescendiente.

Por otro lado, conversando con las entrevistadas, llegamos a la pregunta sobre las razones por las cuales migran de su lugar de origen a otras ciudades. Una de las razones es la búsqueda de oportunidades para el mejoramiento de la calidad de vida, aunque no aunque vale la pena aclarar que no es la razón exclusiva por la que lo hacen, y esto lo afirmó la Mujer Adulta 1 al decir que su proceso fue exclusivamente por seguir a sus familiares más significativos y por la ausencia de uno de ellos, de quien ella se apoyaba para suplir sus necesidades básicas; muy al contrario de la Mujer Adulta 2, para quien la posibilidad de contar con una familia extensa le permitió desplazarse de ciudad en ciudad de forma temporal en busca de un espacio de socialización y de goce con sus familiares (paseo).

Por esta razón, la migración es definida como un cambio temporal o permanente de residencia que se da en los seres humanos que implica el adaptarse a un cambio de vida, en un entorno social, político y económico diferente, donde pueden ocurrir transformaciones en sus prácticas culturales. Al llegar a un contexto diferente se deben enfrentar situaciones y prácticas que si bien pueden coincidir en algunos aspectos, en otros se alejan completamente, dándose un cambio en sus formas de vida poniendo a prueba su capacidad de resiliencia (Barragán, 2005).

En este mismo orden de ideas, la acción social juega un papel importante en dichas transformaciones al ser una acción educativa que se da a diario, donde las personas son el resultado de la interacción social que han tenido a lo largo de su vida, partiendo de la premisa de que las relaciones sociales no son estables, idénticas o constantes, y por ello, se tiene la opción de someterlas a continuo cambio en donde se dan significados de acuerdo a la interpretación que cada persona les da, es entonces como todas las personas son sometidas a procesos de enculturación, en donde cada una puede reconocer quien es y sus posibilidades de acción, para después reflexionar sobre sus prácticas culturales y decidir transformarlas o conservarlas según su necesidad (Mèlich, 1994).

En consecuencia, la acción social es un proceso formativo, una formación que a diario se expresa de manera más notoria en la comunidad afrodescendiente al migrar de su lugar de origen, esto lo corroboró la Mujer Adulta 2, para quien significó la transformación de algunas de sus costumbres, que con el tiempo ha logrado adaptarse afirmando que, para ella tales cambios han sido para mejorar, ha logrado tener una alimentación más amplia y diversificada, con mayor acceso y facilidad de los alimentos a los que no tenía acceso en su lugar de origen, caso contrario al de la Mujer Adulta 1, quien manifestó:

En el Chocó contaba con muchos sembrados en mi casa como el achote, plátano, papaya, piña, yuca, aliños, ñame, asimismo contábamos con el río al lado donde pescábamos todo tipo de pescado, utilizábamos la atarraya y además mi esposo era cazador por lo que nunca faltaba la carne (Mujer adulta 1). 
De esta manera, el no tener los mismos medios en la ciudad para obtener este tipo de productos, el consumo indiscutiblemente ha bajado debido a que actualmente sus ingresos son directamente proporcionales a lo que pueden consumir.

En este sentido, el cambio social es un hecho social de una comunidad, es con el cambio como se evidencian transformaciones en la cultura al modificarse conocimientos, valores e imaginarios que una comunidad fue acumulando a través de los años. La cultura afrodescendiente es el resultado de una transformación de acuerdo a las condiciones económicas, sociales e históricas a las que ha sido sometida a través de los años; en este caso, las prácticas alimenticias se mueven en la tensión permanente entre la añoranza de lo que había en el lugar de origen, y la adaptación a lo que hay disponible en el lugar donde llegan. Ambos aspectos, podrían verse de manera pedagógica, como elementos que aportan a la constitución de los sujetos. En otras palabras,es aquí como se visibiliza que una persona o comunidad puede transformarse o cambiar al migrar para adaptarse al entorno, dando continuidad a algunos valores, costumbres y tradiciones de la cultura de su lugar de origen.

\section{Discusión.}

Partiendo del tema de interés sobre los cambios dados en las prácticas alimenticias de las familias afrodescendientes de la Comuna Ocho en la ciudad de Medellín cuando han migrado de su lugar de origen por diversos factores personales, grupales, culturales, politicos y/o económicos, se pudo dar cuenta que si bien es cierto, se encontraron investigaciones que abordan las prácticas alimenticias a partir de diversos discursos, tales como la nutrición, la medicina, la antropología, la sociología, entre otros, pero de manera desafortunada para intereses de este trabajo, la pedagogía es poco o nada lo que puede decir con respecto a estos temas en nuestro contexto colombiano.

Por esta razón la búsqueda de la información en cuanto a prácticas alimenticias se dio a partir de investigaciones desarrolladas desde otros campos disciplinares; también fue necesario acudir a diferentes fuentes bibliográficas por fuera del territorio local y nacional, además de retomar información brindada por las familias, quienes a partir de su oralidad, expresaron aspectos que permitieron la comprensión de algunnos aspectos alrededor de sus prácticas alimenticias y las posibles transformaciones al migrar de su lugar de origen a otro.

Ahora bien, en cuanto al concepto de familias afrodescendientes no se encontró insumos suficientes que arrojaran una definición precisa. Sin embargo, cabe mencionar la investigación "Lo divino y lo humano, en el territorio de los afrodescendientes: la representación y sacralización del territorio tradicional" (De La Torre, 2012) de la cual se lograron obtener algunos datos para su construcción y comprensión.

\section{Conclusiones.}

Las transformaciones en las prácticas alimenticias son asumidas en las personas al migrar de su lugar de origen de manera subjetiva, debido a las experiencias y oportunidades de vida que han tenido y las que encuentran en el lugar de llegada. El territorio es un factor determinante en los cambios alimenticios debido a que en su lugar de origen, les permitía 
diversificar sus alimentos, cultivados por ellos mismos, y complementado con otras actividades como la caza y la pesca, no sólo para su consumo sino también generadores de ingresos que posibilitaban mejores recursos económicos. Se logra comprender así, algunos procesos de adaptación dados en las entrevistadas, relacionados con sus prácticas alimenticias en cuanto a la adquisición, elaboración y consumo.

Por otro lado, el alimento deja de ser entendido como esa sustancia alimenticia necesaria para el cuerpo y pasa a ser comprendido como un punto primario de encuentro y reencuentro, que genera espacios de significados y recuerdos para la memoria y la identidad afrocolombiana.

En ese sentido, la cultura afrodescendiente se caracteriza por ser una comunidad donde las prácticas alimenticias son adquiridas de generación en generación, y del mismo modo se transmiten recetas de su tierra en las que las mujeres tienen presente los sentidos, conservando así el recuerdo de los sabores, olores y colores, además, el placer de manipular, organizar, combinar, modificar e inventar a la hora de elaborar su receta.

Finalmente, el estudio de caso permitió ampliar, confrontar e interpretar las diferentes prácticas culturales de las familias afrodescendientes cuando han migrado de sus lugares de origen a un nuevo espacio social.

Si bien, esta investigación no se enfocó en asuntos de género, cabe mencionar que en la práctica cultural de las labores del hogar encomendadas a mujeres, se observó que no son actividades muy arraigadas al género, aunque están claras las labores que debe realizar cada uno de los integrantes de la familia, tal como acontece con los hombres integrantes de las familias entrevistadas realizan labores domésticas aprendidas, ya sea por legado familiar o de manera empírica.

\section{Referencias bibliográficas.}

Alonso, L. E., Criado, E. M., \& Moreno, J. L. (2004). Pierre Bourdieu:las herramientas del sociólogo. Madrid: Fundamentos.

Álvarez , J. H., Pemberty , M. A., Blandón A, M., \& Grajales, M. (2012). Otras prácticas de crianza en algunas culturas étnicas de Colombia: un diálogo intercultural Universidad de San Buenaventura, Medellin, pp.89-10. Revista El Ágora USB, 12(1), 89-102.

Barragán, B. L. (2005). El Caribe y sus comunidades transnacionales en Estados Unidos:los casos de la República Dominicana y Haiti. México: Universidad Autónoma de México.

Bautista, N. P. (2011). Proceso de la investigación cualitativa. Epistemología, metodología y aplicaciones. Bogotá: Manual Moderno.

De Certeau , M., Giard, L., \& Mayol, P. (2006). La invención de lo cotidiano. 2, habitar, cocinar. México: Universidad Iberoamericana, Instituto Tecnológico y de Estudios Superiores de Occidente. 
De la Torre, L. M. (2012). Lo divino y lo humano en el territorio de los afrocolombianos:la representación y la sacralización del territorio tradicional. Medellín: Lasallista.

Geertz, C. (2003). La interpretación de las culturas. Barcelona: Gedisa.

Gobernación de Antioquia. (2006). Familia, relaciones y transformaciones. Medellin: Gobernación de Antioquia.

Kornblit, A. L. (2007). Metodologías cualitativas en ciencias sociales. Modelos y procedimientos de análisis. Buenos Aires: Biblos.

Magallanes, A. B., Reyes, R. A., \& Limón, F. (Enero-Abril de 2005). Nutrición de cuerpo y alma:prácticas y creencias alimentarias durante el embarazo en Tziscao, Chiapas. Nueva Antropología, XIX(64), 131-148.

Mélich, J. C. (1994). Del extraño al cómplice: La educación en la vida cotidiana. Barcelona: Anthropos.

Merlino, A. (2009). Investigación cualitativa en ciencias sociales. Temas, problemas y aplicaciones. Buenos Aires: CENCAGE Learning, Grupo AMÉRICALEE.

Pemberty, A. M., Blandón, A. M., \& Grajales, D. M. (2012). Otras prácticas de crianza en algunas culturas étnicas de Colombia:un diálogo intercultural. El ágora USB.

Tajes, M. (2006). El cuerpo de la emigración y la emigración en el cuerpo:desarraigo y negociación de identidad en la literatura de la emigración española. Berna: Peter Lang S.A.Editorial cientifica internacional.

\section{Nota:}

${ }^{7}$ Este artículo es presentado como producto del proceso investigativo que se llevó a cabo a través del proyecto: "Prácticas de crianza escolares, familiares y comunitarias: un diálogo de saberes con las familias de la Asociación de Mujeres Afrodescendientes de Medellín (AMAM) y de la Fundación Golondrinas de la comuna ocho", aprobado en la convocatoria interna de investigación de la Universidad de San Buenaventura seccional Medellin, y cofinanciado por la Corporación Universitaria Lasallista (CUL) y la Fundación Las Golondrinas, ejecutado entre julio de 2014 y diciembre de 2015. Código del proyecto SIGP: 951-14.01.17. 